\title{
UNIQUENESS THEOREMS FOR CONVOLUTION-TYPE EQUATIONS $\left({ }^{1}\right)$
}

\author{
BY \\ J. S. BYRNES AND D. J. NEWMAN
}

One often finds integral equations of the form

(1) $\lambda F(x)-\int_{S} F(t) K(x-t) d t=G(x)$ for $x \in S$ in the literature.

For example, the standard convolution equation is obtained when $S$ is the whole real line. For $S$ the positive real axis, (1) becomes the Wiener-Hopf equation. In aerodynamics, when $S$ is taken to be $[0,1],(1)$ is known as the "lifting line equation."

Our considerations will involve the question of the uniqueness of a solution $F$ of equation (1) in the following special sense: Conditions on $\lambda$ and $K$, together with class conditions on $K$ and $F$ are sought, which will imply the uniqueness of the solution $F$ of equation (1) for all measurable sets $S$.

For each fixed set $S$, uniqueness is equivalent to the assertion

(2) If $\lambda F(x)=\int_{S} F(t) K(x-t) d t$ for all $x \in S$, then $F(x)=0$ for all $x \in S$.

By defining $F$ to be 0 in the complement of $S$, (2) becomes

(3) $\lambda F(x)=F * K(x)$ for $x \in S, F(x)=0$ for $x \notin S$ implies $F(x) \equiv 0$.

And if we take the logical conjunction of the statements (3) for all measurable sets $S$, we get

(4) If, for each $x$, either $F(x)=0$ or $\lambda F(x)=F * K(x)$, then $F(x) \equiv 0$.

Thus, we seek conditions under which (4) holds.

Although most of our work will be done on the real line $(-\infty, \infty)$ and on the group $Z$ of integers $(-\infty<n<\infty)$, we choose as our general setting an arbitrary locally compact abelian group $G$ with Haar measure $d t$. We deal exclusively with the class conditions $F \in L^{\infty}(G)$ and $K \in L^{1}(G)$.

We denote by $\hat{K}(\xi)$ the Fourier transform of $K$. As $\xi$ varies through the elements of $\hat{G}, \hat{K}(\xi)$ traces out a point set in the complex plane, which we call $C_{K}$. The closed convex hull of $C_{K}$ will be called $H_{K}$.

We begin by proving several theorems establishing sufficient conditions for (4) to hold. The basic assumption in these theorems is that $\lambda \notin H_{K}$. If the group $G$ happens to be compact, this is all that is necessary. However, when $G$ is not compact our proofs require added restrictions on $F$ and $K$. The question of whether the theorems are true when we only assume our class conditions on $F$ and $K$ is left open. It appears to be an extremely difficult one.

Received by the editors July $17,1967$.

( ${ }^{1}$ ) This work was part of the first author's doctoral thesis and was supported by United States Steel. Both authors were partially supported by National Science Foundation Grant GP 4391. 
From now on we normalize by setting $\lambda=1$. The hypothesis of (4) that for each $x$, either $F(x)=0$ or $\lambda F(x)=F * K(x)$ can be stated

(5) $(F(x))^{-}[F(x)-F * K(x)] \equiv 0$ where $(F(x))^{-}$denotes the complex conjugate of $F(x)$.

Although the following theorem appears in [3, pp. 630-631], the proof provides the motivation for following results, so we present it for the sake of completeness.

THEOREM I. Suppose that $G$ is a compact group, that (5) holds, and that $1 \notin H_{K}$. Then $F(x) \equiv 0$.

Proof. By (5) we have

$$
\int_{G}(F(x))^{-} F(x) d x-\int_{G}(F(x))^{-} F * K(x) d x=0 .
$$

By the compactness of $G, F$ and $F * K$ are both in $L^{1} \cap L^{2}(G)$. Therefore, Parseval's formula can be applied to (6), and we obtain

$$
\int_{\hat{G}}|\hat{F}(\xi)|^{2} d \xi=\int_{\hat{G}}(\hat{F}(\xi))^{-}(F * K)^{\wedge}(\xi) d \xi=\int_{\hat{G}}|\hat{F}(\xi)|^{2} \hat{R}(\xi) d \xi .
$$

Thus, unless $\hat{F}(\xi)$ is zero almost everywhere (i.e., $F(x) \equiv 0$ ), we have

$$
1=\frac{\int_{\hat{G}}|\hat{F}(\xi)|^{2} \hat{K}(\xi) d \xi}{\int_{\hat{G}}|\hat{F}(\xi)|^{2} d \xi}
$$

which shows that $1 \in H_{K}$, contradicting the hypothesis.

Note that the assumption that $G$ was compact was only needed to insure that $F$ and $F * K$ were both in $L^{1} \cap L^{2}(G)$. Note also that, since $F \in L^{\infty}(G)$ and $K \in$ $L^{1}(G), F \in L^{1}(G)$ implies that $F$ and $F * K$ are both $L^{1} \cap L^{2}(G)$. Thus we have

Corollary I.1. Suppose that $F \in L^{1}(G)$, that (5) holds, and $1 \notin H_{K}$. Then $F(x) \equiv 0$.

However, the most interesting cases occur when $F \notin L^{1}(G)$, so that Parseval's formula need not apply. If $G$ is the real line $(-\infty, \infty)$ we have

THEOREM II. Suppose that (5) holds, that $1 \notin H_{K}$, and that for two nonnegative constants $\alpha$ and $\beta$ we have

(i) $x^{\alpha} K(x) \in L^{1}(-\infty, \infty)$.

(ii) $x^{\beta} F(x) \in L^{\infty}(-\infty, \infty)$.

(iii) $\alpha+2 \beta \geqq 1$.

Then $F(x) \equiv 0$.

Proof. $1 \notin H_{K}$ implies the existence of a complex number $\gamma$ such that

$$
\operatorname{Re}[\gamma(1-\hat{K}(\xi))] \geqq 1, \quad-\infty<\xi<\infty .
$$

For any $\varepsilon>0$, and $a>0$, we can choose $a_{\varepsilon}>a$ such that

$$
\int_{|t|>a}|t|^{\alpha}|K(t)| d t<\varepsilon
$$


Let $V$ denote the set $\left(-a_{\varepsilon}, a_{\varepsilon}\right)$, and $V^{n}$ the set $\left(-n a_{\varepsilon}, n a_{\varepsilon}\right)$. Throughout this proof, $C$ will denote a positive constant, independent of $\varepsilon, a$ and $n$ and not necessarily the same each time it is used. We define, for some $n>1$, a function $f$ by

$$
f=F \text { in } V^{n}, f=0 \text { outside } V^{n} \text {. }
$$

Thus $f$ has compact support and is bounded, so that

$$
f \in L^{1} \cap L^{2} .
$$

We define a function $g$ by

$$
g=|f|^{2}-\bar{f}(f * K)
$$

By (9), we certainly have that

$$
g=0 \text { outside } V^{n}
$$

We wish to estimate $g$ inside $V^{n}$.

$$
\begin{aligned}
g(x) & =|f(x)|^{2}-(f(x))^{-}(f * K(x))=|F(x)|^{2}-(F(x))^{-}(f * K(x)) \\
& =|F(x)|^{2}-(F(x))^{-}(F * K(x))+(F(x))^{-}(F * K(x)-f * K(x)) \\
& =(F(x))^{-} \int_{-\infty}^{\infty}[F(x-t)-f(x-t)] K(t) d t \quad \text { for } x \in V^{n} .
\end{aligned}
$$

Finally, since $x-t \in V^{n} \Rightarrow F(x-t)=f(x-t)$, we get

$$
g(x)=(F(x))^{-} \int_{x-t \notin V^{n}} F(x-t) K(t) d t \text { for } x \in V^{n} .
$$

Now, $x \in V^{n-1}$ and $x-t \notin V^{n} \Rightarrow t \notin V$, so that by (ii) and (8),

$$
\begin{aligned}
\left|\int_{x-t \notin V^{n}} F(x-t) K(t) d t\right| & \leqq \frac{C}{n^{\beta} a_{\varepsilon}^{\beta}} \int_{t \notin V} \frac{1}{|t|^{\alpha}}|t|^{\alpha}|K(t)| d t \\
& \leqq \frac{C \varepsilon}{n^{\beta} a_{\varepsilon}^{\alpha+\beta}} \text { for } x \in V^{n-1} . \\
\int_{V^{n-1}} g(x) d x & =\int_{-1}^{1} g(x) d x+\int_{|x|>1, x \in V^{n-1}} g(x) d x
\end{aligned}
$$

so that, combining (ii), (13), and (14) we get

$$
\begin{aligned}
\left|\int_{V^{n-1}} g(x) d x\right| & \leqq \frac{C \varepsilon}{n^{\beta} a_{\varepsilon}^{\alpha+\beta}}\left[1+\int_{1}^{n a_{\varepsilon}} \frac{C}{x^{\beta}}\right] \\
& \leqq C \varepsilon n^{1-2 \beta} a_{\varepsilon}^{1-\alpha-2 \beta} .
\end{aligned}
$$

Now, (iii) and (15) certainly imply

$$
\left|\int_{V^{n-1}} g(x) d x\right| \leqq C n \varepsilon .
$$


Letting $\Delta=V^{n}-V^{n-1}, \Delta_{1}=V^{n+1}-V^{n}$, and $\Delta_{2}=V^{n+1}-V^{n-1}$, it remains to estimate $g(x)$ for $x \in \Delta$. By (13)

(17) $g(x)=(F(x))^{-} \int_{x-t \notin V^{n}, t \notin V} F(x-t) K(t) d t+(F(x))^{-} \int_{x-t \notin V^{n}, t \in V} F(x-t) K(t) d t$.

$$
\left|(F(x))^{-} \int_{x-t \notin V^{n}, t \notin V} F(x-t) K(t) d t\right| \leqq \frac{C \varepsilon}{a_{\varepsilon}^{\alpha+2 \beta}} \text { for } x \in \Delta,
$$

so that

$$
\left|\int_{\Delta}(F(x))^{-} \int_{x-t \notin V^{n}, t \notin V} F(x-t) K(t) d t d x\right| \leqq C \varepsilon .
$$

Note that $x \in \Delta, t \in V$ implies $x-t \in V^{n+1}$. Therefore,

$$
\begin{aligned}
\mid \int_{\Delta}(F(x))^{-} \int_{x-t \notin V^{n}, t \in V} F(x-t) & K(t) d t d x \mid \\
& \leqq \iint_{x \in \Delta, t \in V, x-t \in \Delta_{1}}|F(x)||F(x-t)||K(t)| d x d t \\
& \leqq \iint_{x \in \Delta_{2}, x-t \in \Delta_{2}, t \in V}|F(x)||F(x-t)||K(t)| d x d t .
\end{aligned}
$$

For each fixed $t$, the Schwarz inequality yields

$$
\int_{x \in \Delta_{2}, x-t \in \Delta_{2}}|F(x)||F(x-t)| d x \leqq \int_{\Delta_{2}}|F(x)|^{2} d x .
$$

Combining (19) and (20), we get

$$
\begin{aligned}
\left|\int_{\Delta}(F(x))-\int_{x-t \notin V^{n}, t \in V} F(x-t) K(t) d t d x\right| & \\
& \leqq \int_{-\infty}^{\infty}|K(t)| d t \cdot \int_{\Delta_{2}}|F(t)|^{2} d t=M \int_{\Delta_{2}}|F|^{2},
\end{aligned}
$$

where $M=\int_{-\infty}^{\infty}|K|$. Finally, combining (16), (17), (18) and (21) yields

$$
\left|\int_{V^{n}} g(x) d x\right| \leqq C n \varepsilon+M \int_{\Delta_{2}}|F|^{2} .
$$

By virtue of (11) and (12), (22) can be written

$$
\left.\left|\int_{G}\right| f\right|^{2}-\left.\bar{f}(f * K)\left|\leqq C n \varepsilon+M \int_{\Delta_{2}}\right| F\right|^{2} .
$$

By (10) Parseval's formula can be applied, and (23) yields

$$
\left.\left.\left|\int_{\hat{G}}\right| \hat{f}\right|^{2}(1-\hat{R})\left|\leqq C n \varepsilon+M \int_{\Delta_{2}}\right| F\right|^{2}
$$

By virtue of (7), (24) shows that

$$
\int_{\hat{G}}|\hat{f}|^{2} \leqq|\gamma| C n \varepsilon+M|\gamma| \int_{\Delta_{2}}|F|^{2}
$$


and applying Parseval's formula once more gives

$$
\int_{G}|f|^{2}=\int_{V^{n}}|F|^{2} \leqq C n \varepsilon+M|\gamma| \int_{\Delta_{2}}|F|^{2}
$$

Since $\Delta_{1} \subset \Delta_{2}$, we certainly have

$$
\int_{\Delta_{1}}|F|^{2} \leqq \int_{\Delta_{2}}|F|^{2}
$$

Adding inequalities (26) and (27) yields

$$
\int_{V^{n+1}}|F|^{2} \leqq C n \varepsilon+(M|\gamma|+1) \int_{\Delta_{2}}|F|^{2}
$$

and by a rearrangement of (28) we get

$$
\int_{V^{n-1}}|F|^{2} \leqq C n \varepsilon+\delta \int_{V^{n+1}}|F|^{2} \quad \text { where } \delta=\frac{M|\gamma|}{M|\gamma|+1}<1 .
$$

Repeated application of (29) yields

$$
\begin{aligned}
\int_{V}|F|^{2} & \leqq 2 C \varepsilon \sum_{k=1}^{n} k \delta^{k-1}+\delta^{n} \int_{V^{2 n+1}}|F|^{2} \\
& <2 C \varepsilon \sum_{k=1}^{\infty} k \delta^{k-1}+\delta^{n} \int_{V^{2 n+1}}|F|^{2} \leqq C \varepsilon+C n \delta^{n} a_{\varepsilon} .
\end{aligned}
$$

If we now let $n \rightarrow \infty$, we get

$$
\int_{V}|F|^{2} \leqq C \varepsilon
$$

Recalling that $a_{\varepsilon}>a$, (31) shows that

$$
\int_{-a}^{a}|F|^{2} \leqq C \varepsilon
$$

But $\varepsilon$ and $a$ are arbitrary, so (32) yields

$$
\int_{G}|F|^{2}=0
$$

Finally, (33) says that $F=0$ almost everywhere in $G$. But this implies that $F * K \equiv 0$, and (5) now tells us that $F \equiv 0$, which is the desired result.

We now give several corollaries of Theorem II. The first three of these are immediate consequences of the theorem.

Corollary II.1. Let $G$ be the real line $(-\infty, \infty)$, and suppose that (5) holds, that $1 \notin H_{K}$, and that $F(x)=O\left(|x|^{-1 / 2}\right)$ as $x \rightarrow \pm \infty$. Then $F \equiv 0$.

Corollary II.2. Let $G$ be the real line $(-\infty, \infty)$, and suppose that (5) holds, that $1 \notin H_{K}$, and that $x K(x) \in L^{1}(-\infty, \infty)$. Then $F \equiv 0$. 
Corollary II.3 (D. J. Newman). Let $G$ be the real line, and suppose that (5) holds, that $1 \notin H_{K}$, and that $K$ has compact support. Then $F \equiv 0$.

COROLlaRY II.4. Let $G$ be the real line, and suppose that (5) holds and that $1 \notin H_{K}$. Then $|F|^{2} \rightarrow 0$ on the average.

Proof. We proceed precisely as we did in the proof of Theorem II, taking $\alpha=\beta$ $=0$. Thus estimate (16) becomes

$$
\left|\int_{V^{n-1}} g(x) d x\right| \leqq C n a_{\varepsilon} \varepsilon
$$

and estimate (18) becomes

$$
\left|\int_{\Delta}(F(x))^{-} \int_{x-t \notin V^{n}, t \in V} F(x-t) K(t) d t d x\right| \leqq C a_{\varepsilon} \varepsilon .
$$

Estimate (21) remains the same, so that (22) is now

$$
\left|\int_{V^{n}} g(x) d x\right| \leqq C n a_{\varepsilon} \varepsilon+M \int_{\Delta_{2}}|F|^{2} .
$$

From here we continue exactly as in the theorem, and end up with $\int_{V}|F|^{2} \leqq C a_{\varepsilon} \varepsilon$ or

$$
\frac{\int_{V}|F|^{2}}{m(V)} \leqq C \varepsilon, \quad \text { where } m(V)=\text { the measure of } V
$$

Thus

$$
\lim _{m(V) \rightarrow \infty} \frac{\int_{V}|F|^{2}}{m(V)}=0, \quad \text { or }|F|^{2} \rightarrow 0
$$

on the average.

Corollary II.5. Let $G$ be the real line, and suppose that (5) holds and that $1 \notin H_{K}$. Then $|F| \rightarrow 0$ on the average.

Proof. By (34) and the Schwarz inequality, we have

$$
\int_{V}|F| \leqq\left(\int_{V}|F|^{2}\right)^{1 / 2}(m(V))^{1 / 2} \leqq m(V)(C \varepsilon)^{1 / 2}
$$

so that

$$
\lim _{m(V) \rightarrow \infty} \frac{\int_{V}|F|}{m(V)}=0, \quad \text { or }|F| \rightarrow 0
$$

on the average.

Corollary II.6. Let $G$ be $E^{N}$ (i.e., $N$ dimensional Euclidean space) for $N \geqq 1$. Suppose that (5) holds, that $1 \notin H_{K}$, and that for two nonnegative constants $\alpha$ and $\beta$ we have 
(i) $x^{\alpha} K(x) \in L^{1}(G)$ where $x=\left(x_{1}, x_{2}, \ldots, x_{N}\right)$.

(ii) $F\left(x_{1}, x_{2}, \ldots, x_{N}\right)=O\left(1 /\left|x_{1} x_{2} \cdots x_{N}\right|^{\beta}\right)$ as $x_{i} \rightarrow \pm \infty, 1 \leqq i \leqq N$.

(iii) $\alpha+2 N \beta \geqq N$.

Then $F \equiv 0$.

Proof. Once again we proceed as in Theorem II. Our set $V$ becomes a compact subset of $E^{N}$, symmetric about the origin, with sides $2 a_{\varepsilon}$. The auxiliary functions $f$ and $g$ are defined as before, and estimate (16) becomes

$$
\left|\int_{V^{n-1}} g(x) d x\right| \leqq C n^{N} \varepsilon
$$

which changes estimate (22) to

$$
\left|\int_{V^{n}} g(x) d x\right| \leqq C n^{N} \varepsilon+M \int_{\Delta_{2}}|F|^{2} .
$$

This requires us to change (30) to

$$
\begin{aligned}
\int_{V}|F|^{2} & \leqq 2^{N} C \varepsilon \sum_{k=1}^{n} k^{N} \delta^{k-1}+\delta^{n} \int_{V^{2 n+1}}|F|^{2} \\
& <2^{N} C \varepsilon \sum_{k=1}^{\infty} k^{N} \delta^{k-1}+\delta^{n} \int_{V^{2 n+1}}|F|^{2} \leqq C \varepsilon+C n \delta^{n} a_{\varepsilon}
\end{aligned}
$$

which is exactly what we had before. The remainder of the proof is the same, and we conclude that $F \equiv 0$.

Although the setting for Theorem II and its corollaries was taken to be Euclidean space, all of the proofs apply equally as well to the group $Z$ of integers, or in the case of Corollary II.6, to the group $Z^{N}$ of lattice points in $E^{N}$. Thus we have

COROLlary II.7. If we take our group $G$ to be $Z$, then Theorem II and Corollaries II.1-II.5 are valid. If our group is $Z^{N}$, then Corollary II.6 is valid.

Furthermore, let us observe that if $H$ is any compact group and $G$ is one of the above groups, the proofs of the above corollaries apply immediately to $G \oplus H$, the direct sum of $G$ and $H$ (just take our set $V$ so that it contains $H$ ). Combining this remark with Corollaries II.6 and II.7, we get

Corollary II.8. Let $G$ be $E^{N} \oplus Z^{M} \oplus H$, where $N \geqq 0, M \geqq 0$, and $H$ is $a$ compact group. Suppose that (5) holds, that $1 \notin H_{K}$, and that for two nonnegative constants $\alpha$ and $\beta$ we have

(i) $x^{\alpha} K(x) \in L^{1}(G)$ where $x=\left(x_{1}, \ldots, x_{N}, n_{1}, \ldots, n_{M}, y\right)$.

(ii) As $x_{i} \rightarrow \pm \infty, 1 \leqq i \leqq N$ and $n_{j} \rightarrow \pm \infty, 1 \leqq j \leqq M$,

$$
F\left(x_{1}, x_{2}, \ldots, x_{N}, n_{1}, n_{2}, \ldots, n_{M}, y\right)=O\left(\frac{1}{\left|x_{1} x_{2} \cdots x_{N} n_{1} n_{2} \cdots n_{M}\right|^{\beta}}\right) .
$$

(iii) $\alpha+2(N+M) \beta \geqq N+M$.

Then $F \equiv 0$. 
A locally compact abelian group $G$ is called compactly generated if it contains a compact subset $C$ for which the subgroup generated by $C$ is $G$. It is known that every locally compact, compactly generated abelian group $G$ is topologically isomorphic with $E^{N} \oplus Z^{M} \oplus H$ where $H$ is compact [2, p. 90]. If we let the dimension $d$ of such a group $G$ be $N+M$, then we get from Corollary II. 8 and the above remarks:

Corollary II.9. Let $G$ be compactly generated with dimension $d$. Suppose that (5) holds, that $1 \notin H_{K}$, and that for two nonnegative constants $\alpha$ and $\beta$ we have

(i) $x^{\alpha} K(x) \in L^{1}(G)$ where $x=\left(x_{1}, x_{2}, \ldots, x_{d}, y\right)$.

(ii) As $x_{i} \rightarrow \pm \infty, 1 \leqq i \leqq d$,

$$
F\left(x_{1}, x_{2}, \ldots, x_{d}, y\right)=O\left(\frac{1}{\left|x_{1} x_{2} \cdots x_{d}\right|^{\beta}}\right) .
$$

(iii) $\alpha+2 d \beta \geqq d$.

Then $F \equiv 0$.

This appears to be the strongest theorem of this generality that can be proven utilizing the methods of Theorem II. In the more general case of an arbitrary locally compact abelian group, it seems that the best result one can derive from these methods is Corollary II.3 [3, pp. 630-634].

By altering our approach, we are able to improve upon Corollary II.2. In particular, we have:

TheOREM III. Let $G$ be the real line $(-\infty, \infty)$, and suppose that (5) holds, that $1 \notin H_{K}$, and that $|x|^{1 / 2} K(x) \in L^{1}(-\infty, \infty)$. Then $F \equiv 0$.

Proof. (We are grateful to L. Carleson for suggesting the idea of this proof.) We define a function $F_{n}$ by

$$
F_{n}(x)=F(x), \quad 0 \leqq|x|<n \quad \text { and } \quad F_{n}(x)=0, \quad|x| \geqq n,
$$

and another function $g$ by

$$
g=\bar{F}_{n}\left(F_{n}-F_{n} * K\right)
$$

We can assume that

$$
\int_{-n}^{n}|F|^{2}=\int_{-\infty}^{\infty}\left|F_{n}\right|^{2} \text { goes to } \infty \text { as } n \rightarrow \infty
$$

since otherwise we can use Fourier transforms directly to prove the theorem. Combining (5), (35), and (36) we get that for $|x|<n$,

$$
\begin{aligned}
g(x) & =\bar{F}\left(F-F_{n} * K\right)=\bar{F}(F-F * K)+\bar{F}\left(F * K-F_{n} * K\right) \\
& =\bar{F}\left(F * K-F_{n} * K\right) .
\end{aligned}
$$


Since obviously $g=0$ for $|x| \geqq n$, (38) yields

$$
\begin{aligned}
\int_{-\infty}^{\infty} g(x) d x & =\int_{-\infty}^{\infty}\left|F_{n}(x)\right|^{2} d x-\int_{-\infty}^{\infty}\left(F_{n}(x)\right)^{-} F_{n} * K(x) d x \\
& =\int_{-n}^{n}(F(x))^{-} \int_{|x-t|>n} F(x-t) K(t) d t d x .
\end{aligned}
$$

$F_{n}$ has compact support and is bounded, so that $F_{n} \in L^{1} \cap L^{2}$. Therefore, the Parseval formula can be applied to (39) and we get

$$
\int_{-\infty}^{\infty}\left|\hat{F}_{n}\right|^{2}=\int_{-\infty}^{\infty}\left|\hat{F}_{n}\right|^{2} R+\int_{-n}^{n}(F(x))^{-} \int_{|x-t|>n} F(x-t) K(t) d t d x .
$$

By the Schwarz inequality we get

$$
\begin{aligned}
\mid \int_{-n}^{n}(F(x))-\int_{|x-t|>n} F(x-t) & K(t) d t d x \mid \\
& \leqq\left(\int_{-n}^{n}|F|^{2}\right)^{1 / 2}\left(\int_{-n}^{n}\left(\int_{|x-t|>n}|K(t)| d t\right)^{2} d x\right)^{1 / 2} .
\end{aligned}
$$

Since $|x|^{1 / 2} K(x) \in L^{1}(-\infty, \infty)$ we see that

$$
\begin{aligned}
& \int_{-n}^{n}\left(\int_{|x-t|>n}|K(t)| d t\right)^{2} d x \\
&=\int_{-n}^{n} \int_{n+x}^{\infty} \int_{n+x}^{\infty}|K(s)||K(t)| d t d s d x \\
&+\int_{-n}^{n} \int_{-\infty}^{-n+x} \int_{n+x}^{\infty}|K(s)||K(t)| d t d s d x \\
&+\int_{-n}^{n} \int_{n+x}^{\infty} \int_{-\infty}^{-n+x}|K(s)||K(t)| d t d s d x \\
&+\int_{-n}^{n} \int_{-\infty}^{-n+x} \int_{-\infty}^{-n+x}|K(s)||K(t)| d t d s d x \\
&= \int_{0}^{\infty} \int_{0}^{\infty} \min (s, t)|K(s)||K(t)| d s d t+3 \text { similar terms } \\
& \leqq \int_{0}^{\infty} \int_{0}^{\infty}(s t)^{1 / 2}|K(s)||K(t)| d s d t+3 \text { similar terms } \\
&= \int_{0}^{\infty} \sqrt{ } s|K(s)| d s \int_{0}^{\infty} \sqrt{ } t|K(t)| d t+3 \text { similar terms = } O(1) .
\end{aligned}
$$

Recalling that $\int_{-\infty}^{\infty}\left|\hat{F}_{n}\right|^{2}=\int_{-\infty}^{\infty}\left|F_{n}\right|^{2}=\int_{-n}^{n}|F|^{2}$ goes to $\infty$ as $n \rightarrow \infty$, we can combine (40), (41) and (42) to get

$$
1=\frac{\int_{-\infty}^{\infty}\left|\hat{F}_{n}\right|^{2} R}{\int_{-\infty}^{\infty}\left|\hat{F}_{n}\right|^{2}}+o(1)
$$

which says that $1 \in H_{K}$, contradicting the hypothesis. 
NoTE. The hypothesis $|x|^{1 / 2} K \in L^{1}$ can obviously be replaced by

$$
\int_{|t|>x} K(t) d t \in L^{2}(-\infty, \infty)
$$

Corollary III.1. Let $G$ be the real line $(-\infty, \infty)$, and suppose that equation (5) holds, that $1 \notin H_{K}$, and that

$$
\lim _{n \rightarrow \infty} \frac{\max _{|t|>n}|F(t)| \cdot \int_{-n}^{n}|F(x)| d x}{\int_{-n}^{n}|F(x)|^{2} d x}=0 .
$$

Then $F \equiv 0$.

Proof. Since $K \in L^{1}$, we have, with $M=\int_{-\infty}^{\infty}|K|$

$$
\left|\int_{-n}^{n} F(x) \int_{|t| \geqq n} F(t) K(x-t) d t d x\right| \leqq M \max _{|t| \geqq n}|F(t)| \int_{-n}^{n}|F(x)| d x .
$$

Dividing (40) by $\int_{-\infty}^{\infty}\left|\hat{F}_{n}\right|^{2}=\int_{-n}^{n}|F|^{2}$ and using (37), (44), and (45) we get equation (43), which again says that $1 \in H_{K}$.

Note that if $F(x)=O\left(|x|^{-1 / 2}\right)$ as $x \rightarrow \pm \infty$ we can use the Schwarz inequality to get

$$
\max _{|t \geqq n|}|F(t)| \cdot \int_{-n}^{n}|F(x)| d x=O\left(\left(\int_{-n}^{n}|F|^{2}\right)^{1 / 2}\right) .
$$

Combining (46) with Corollary III.1, we see that we have a new proof of Corollary II.1.

Once again we observe that the proofs of Theorem III and Corollary III.1 would apply equally as well to the group $Z$ of integers. Thus we have

Corollary III.2. If we take $G$ to be $Z$, then Theorem III and Corollary III.1 are valid.

We now turn our attention to the question of a converse to the above theorems. In particular we ask whether the truth of (4) implies that $\lambda \notin H_{K}$. If $K$ happens to be Hermitian (i.e., $K(-t)=(K(t))^{-}$) we have the following result, due to D. J. Newman (see [3, p. 630] where a nice proof is presented).

THEOREM IV. Suppose that (4) holds, that $K$ is Hermitian, that $\lambda \neq 0$ and that $\hat{G}$ is connected. Then $\lambda \notin H_{K}$.

For the remainder of our work we will consider as our group $G$ the group $Z$ of integers. In addition, we will restrict our attention to a kernel $K$ of a very specific type, namely one with just two mass points. Thus $K$ will be a complex sequence $\left\{c_{n}\right\}$ where

$$
\begin{aligned}
c_{n} & =\alpha, & & n=n_{1}, \\
& =\beta, & & n=n_{2}, \\
& =0, & & n \neq n_{1}, n_{2} .
\end{aligned}
$$


Our function $F$ becomes a bounded sequence $\left\{a_{n}\right\}$ of complex numbers, and our problem is to find conditions under which the following statement holds:

(47) If, for each $n$, either $a_{n}=0$ or $\lambda a_{n}=\alpha a_{n-n_{1}}+\beta a_{n-n_{2}}$, and if $\left\{a_{n}\right\}$ is bounded, then $a_{n} \equiv 0$.

Corollary II.3 certainly tells us that $\lambda \notin H_{K}$ implies (47). However, we shall soon see that even with the simple kernel described above, the truth of (47) need not imply that $\lambda \notin H_{K}$.

We begin by showing that for a subclass of the kernels of the type described above, (47) is equivalent to $\lambda \notin H_{K}$. Namely,

THEOREM V. Suppose one of the two mass points of the kernel $K=\left\{c_{n}\right\}$ is the origin. Then (47) implies that $\lambda \notin H_{K}$.

\section{Proof.}

$$
\begin{aligned}
c_{n} & =\alpha, & & n=0, \\
& =\beta, & & n=n_{2}, \\
& =0, & & n \neq 0, n_{2},
\end{aligned}
$$

so that

$$
R(\xi)=\sum_{n=-\infty}^{\infty} c_{n} e^{-i n \xi}=\alpha+\beta e^{-i n_{2} \xi}, \quad 0 \leqq \xi<2 \pi .
$$

Obviously, $C_{K}=\{z:|z-\alpha|=|\beta|\}$ and $H_{K}=\{z:|z-\alpha| \leqq|\beta|\}$. So, for each $\lambda \in H_{K}$, we must produce a bounded sequence $\left\{a_{n}\right\}$ which is not identically 0 and which satisfies the hypothesis of (47). Such a sequence is as follows: Let $a_{0}=1, a_{-k n_{2}}$ $=((\lambda-\alpha) / \beta)^{k}$ for $k=1,2,3, \ldots$ and $a_{n}=0$ for all other $n$. It is obvious that this sequence has the required properties.

We now consider (47) with $\alpha$ and $\beta$ real, and we normalize by setting $\beta=-1$. Furthermore, we set $n_{1}=1$ and $n_{2}=k>1$. Thus (47) becomes:

(48). If, for each $n$, either $a_{n}=0$ or $\lambda a_{n}=\alpha a_{n-1}-a_{n-k}$ and if $\left\{a_{n}\right\}$ is bounded, then $a_{n} \equiv 0$.

Under these circumstances, we are again able to produce our desired converse.

Theorem VI. Suppose that in (48), $|\alpha| \geqq k^{2}$. Then, if we consider only real $\lambda$, (48) implies that $\lambda \notin H_{K}$.

Proof. $\hat{K}(\xi)=\alpha e^{-i \xi}-e^{-i k \xi}, 0 \leqq \xi<2 \pi$. $\operatorname{Re} \hat{K}(\xi)=\alpha \cos \xi-\cos k \xi$. Taking the derivative of $\operatorname{Re} \hat{K}(\xi)$ to find its extreme values, we get $-\alpha \sin \xi+k \sin k \xi=0$. Thus, either $\sin \xi=0$ or $\alpha=k \sin k \xi / \sin \xi$. But $|k \sin k \xi / \sin \xi|<k^{2}$ if $\sin \xi \neq 0$. Since by hypothesis $|\alpha| \geqq k^{2}$, the extreme values of $\operatorname{Re} \hat{K}(\xi)$ must occur at $\xi=0$ and $\xi=\pi$. $\hat{K}(0)=\alpha-1$ and $\hat{K}(\pi)=-\alpha-(-1)^{k}$.

Since the extreme values of $\operatorname{Re} \hat{K}(\xi)$ are taken on by $\hat{K}(\xi)$, a real $\lambda$ will be in $H_{K}$ if and only if $\lambda$ is between $\alpha-1$ and $-\alpha-(-1)^{k}$. So, for each $\lambda$ in that range, we must produce a bounded sequence $\left\{a_{n}\right\}$ which is not identically 0 and which 
satisfies the hypothesis of (48). If $\lambda=0$, we can take $a_{0}=1$ and $a_{n}=0$ for $n \neq 0$. If $\lambda=\alpha-1$, we can take $a_{n} \equiv 1$. If $\lambda=-\alpha-(-1)^{k}$, we can take $a_{n}=(-1)^{n}$. In order to deal with other values of $\lambda$, we break down the problem into four cases. The proofs of the four cases are almost identical, so we present a typical one. Suppose $\alpha \geqq k^{2}$ and $0<\lambda<\alpha-1$. Let $f(t)=\lambda t^{k}-\alpha t^{k-1}+1$. $f(1)=\lambda-\alpha+1<0$. As $t \rightarrow+\infty$, $f(t) \rightarrow+\infty$. Thus, $f(t)$ has at least one zero in $(1, \infty)$. Let the smallest one be $t_{0}$. Then we can let our sequence be $a_{n}=0$ for $n>0$ and $a_{n}=t_{0}^{n}$ for $n \leqq 0$.

To conclude, we present several examples for which (4) holds but $\lambda \in H_{k}$. As stated above, our group will be $Z$ and our kernel $K$ will have two mass points. In particular, $K$ will be $\left\{c_{n}\right\}$ where

$$
\begin{aligned}
c_{n} & =\alpha, & & n=1, \\
& =\beta, & & n=2, \\
& =0, & & n \neq 1,2 .
\end{aligned}
$$

Thus, $\hat{K}(\xi)=\alpha e^{-i \xi}+\beta e^{-2 i \xi}, 0 \leqq \xi<2 \pi$. (4) becomes

(49) If, for each $n$, either $a_{n}=0$ or $\lambda a_{n}=\alpha a_{n-1}+\beta a_{n-2}$, and if $\left\{a_{n}\right\}$ is bounded, then $a_{n} \equiv 0$.

EXAMPLE i. We let $\beta=-1,-4<\alpha \leqq-3$, and we consider only positive $\lambda$.

$$
\operatorname{Re} \hat{K}(\xi)=\alpha \cos \xi-\cos 2 \xi \text {. }
$$

For $\operatorname{Re} \hat{K}(\xi)$ to have a maximum, we need

$$
-\alpha \sin \xi+2 \sin 2 \xi=0 .
$$

Thus, either $\sin \xi=0$ or

(50) $\cos \xi=\alpha / 4$.

$\operatorname{Re} \hat{K}(0)=\alpha-1$ which is obviously the minimum of $\operatorname{Re} \hat{K}(\xi)$.

(51) $\operatorname{Re} \hat{K}(\pi)=-\alpha-1$. If (50) holds, we get $\operatorname{Re} \hat{K}(\xi)=\alpha^{2} / 8+1$.

Furthermore, since both points $\alpha^{2} / 8+1 \pm i(\alpha / 2)\left(1-\alpha^{2} / 16\right)^{1 / 2}$ are in $C_{K}$, we certainly have that

(52) $\alpha^{2} / 8+1 \in H_{K}$.

Also, for $\alpha \neq-4,(\alpha+4)^{2} / 8=\alpha^{2} / 8+\alpha+2>0$ or

(53) $\alpha^{2} / 8+1>-\alpha-1$.

Combining (51), (52), and (53) we see that, when considering only positive $\lambda$, (54) $\lambda \in H_{K}$ if and only if $\lambda \leqq \alpha^{2} / 8+1$.

However we will show that, when considering positive $\lambda$,

(55) A bounded sequence $\left\{a_{n}\right\}$, satisfying the hypothesis of (49) and not identically zero, will exist if and only if $\lambda \leqq-\alpha-1$.

Thus we will have that, for $-\alpha-1<\lambda \leqq \alpha^{2} / 8+1$, (4) holds but $\lambda \in H_{K}$. It remains to prove (55).

Let us consider the form of the sequence which we are trying to decide the existence of. We know that for each $n$, either $a_{n}=0$ or $\lambda a_{n}=\alpha a_{n-1}-a_{n-2}$. Obviously, if $a_{k}=a_{k+1}=0$ for any integer $k(-\infty<k<\infty)$, then $a_{n}=0$ for all $n \geqq k$. Thus, in 
order to have a sequence of the required form which is not identically zero, there must exist an integer $N(-\infty<N<\infty)$ such that for any $k<N,\left|a_{k}\right|+\left|a_{k+1}\right|>0$.

In the equation $\lambda a_{n}-\alpha a_{n-1}+a_{n-2}=0$ let $a_{n}=t^{n}$. Dividing the equation by $t^{n-2}$, we get

(56) $\lambda t^{2}-\alpha t+1=0$.

Solving for $t$, we get

(57) $t=\left(\alpha \pm\left(\alpha^{2}-4 \lambda\right)^{1 / 2}\right) / 2 \lambda$

and as the most general solution to our equation we get

$$
a_{n}=A\left(\frac{\alpha+\left(\alpha^{2}-4 \lambda\right)^{1 / 2}}{2 \lambda}\right)^{n}+B\left(\frac{\alpha-\left(\alpha^{2}-4 \lambda\right)^{1 / 2}}{2 \lambda}\right)^{n},
$$

where $A$ and $B$ are arbitrary constants. By (54) we know that for $\lambda>\alpha^{2} / 8+1$ (49) is true, so that we need only consider $\lambda \leqq \alpha^{2} / 8+1$. Combining this with $\alpha \leqq-3$, we note that certainly $\alpha^{2}-4 \lambda>0$, so that the square roots occurring in (57) and (58) are real. Thus,

$$
\left|\frac{\alpha+\left(\alpha^{2}-4 \lambda\right)^{1 / 2}}{2 \lambda}\right|<\frac{-\alpha}{2 \lambda}+\frac{\left(\alpha^{2}-4 \lambda\right)^{1 / 2}}{2 \lambda}=\left|\frac{\alpha-\left(\alpha^{2}-4 \lambda\right)^{1 / 2}}{2 \lambda}\right| .
$$

Furthermore, if $\lambda \leqq-\alpha-1$ then

$$
\left|\frac{\alpha-\left(\alpha^{2}-4 \lambda\right)^{1 / 2}}{2 \lambda}\right| \geqq \frac{-\alpha}{2(-\alpha-1)}+\frac{\left(\alpha^{2}+4 \alpha+4\right)^{1 / 2}}{2(-\alpha-1)}=\frac{-\alpha-(\alpha+2)}{2(-\alpha-1)}=1 .
$$

Therefore, if we choose for $n \leqq 0$ the $a_{n}$ given by (58) with $A=0$ and $B=1$, and let $a_{n}=0$ for $n>0$, we get a sequence $\left\{a_{n}\right\}$ which satisfies the hypothesis of (49). This proves the "if" half of (55).

Consider now what happens when $\lambda>-\alpha-1$. By (59),

$$
\left|\frac{\alpha+\left(\alpha^{2}-4 \lambda\right)^{1 / 2}}{2 \lambda}\right|<\left|\frac{\alpha-\left(\alpha^{2}-4 \lambda\right)^{1 / 2}}{2 \lambda}\right|<\frac{-\alpha+\left(\alpha^{2}+4 \alpha+4\right)^{1 / 2}}{2(-\alpha-1)}=1 .
$$

If our sequence $\left\{a_{n}\right\}$ is part of a fixed general solution (i.e., $A$ and $B$ fixed in (58)) beginning from some index $n_{0}<N$ and continuing to $-\infty,(61)$ shows that it must be unbounded. The only remaining possibility is that, as we move left (i.e., the index decreases) from $a_{N}$, the sequence becomes zero an infinite number of times (recall that the sequence does not have two consecutive zeros in this range). If $a_{n}=0$, we are able to change our solution, since the equation $\lambda a_{n}-\alpha a_{n-1}+a_{n-2}$ $=0$ need not hold. However, the change cannot be arbitrary, since $a_{n-1}$ will be determined by $a_{n+1}$.

We will show that around each zero there must be a jump in absolute value (as the index decreases) of more than $\lambda /(-\alpha-1)$. Thus, since $\lambda>-\alpha-1$ and there are an infinite number of jumps, our sequence must again be unbounded.

We can assume, without loss of generality, that the zero occurs at $a_{0}$. Thus, we have the following situation:

(62) $a_{0}=0, a_{-1} \neq 0$, and none of $a_{1}, a_{2}, \ldots, a_{n}$ are 0 , where $n \geqq 1$. 
We wish to show that

(63) $\left|a_{-1}\right|>(\lambda /(-\alpha-1))\left|a_{n}\right|$.

Since $a_{0}=0$ is part of a general solution, we have by (58) $A+B=0$. We can assume that $-A=B=1$.

Also by (58),

$$
a_{-1}=\frac{-2 \lambda}{\alpha+\left(\alpha^{2}-4 \lambda\right)^{1 / 2}}+\frac{2 \lambda}{\alpha-\left(\alpha^{2}-4 \lambda\right)^{1 / 2}}=\left(\alpha^{2}-4 \lambda\right)^{1 / 2} .
$$

Thus, $\left|a_{-1}\right|=\left(\alpha^{2}-4 \lambda\right)^{1 / 2}$. Similarly,

$$
a_{n}=\frac{1}{(2 \lambda)^{n}}\left[-\left(\alpha+\left(\alpha^{2}-4 \lambda\right)^{1 / 2}\right)^{n}+\left(\alpha-\left(\alpha^{2}-4 \lambda\right)^{1 / 2}\right)^{n}\right] .
$$

Since the two terms appearing between the brackets must point in opposite directions, and since the term on the right is of greater magnitude, we have

$$
\left|a_{n}\right|=\frac{1}{(2 \lambda)^{n}}\left[\left(-\alpha+\left(\alpha^{2}-4 \lambda\right)^{1 / 2}\right)^{n}-\left(-\alpha-\left(\alpha^{2}-4 \lambda\right)^{1 / 2}\right)^{n}\right] .
$$

Since we are assuming $\alpha \leqq-3$, we get

$$
-\alpha-2 \geqq 1>1-\frac{1}{(-\alpha-1)^{n}} \text { or } \quad(-\alpha-1)^{n}>\frac{(-\alpha-1)^{n}-1}{-\alpha-2} \text {. }
$$

But $-\alpha-1<\lambda$, so that $\lambda^{n-1}(-\alpha-1)>\left((-\alpha-1)^{n}-1\right) /(-\alpha-2)$. Dividing by $\lambda^{n}$ and adding some extra terms, we get

$$
\frac{2}{(2 \lambda)^{n}} \frac{2^{n}(-\alpha-1)^{n}-2^{n}}{2(-\alpha-2)}<\frac{-\alpha-1}{\lambda} \text {. }
$$

$$
\begin{aligned}
\frac{2}{(2 \lambda)^{n}} \frac{2^{n}(-\alpha-1)^{n}-2^{n}}{2(-\alpha-2)} & =\frac{2}{(2 \lambda)^{n}} \frac{[-\alpha+(\alpha+2)]^{n}-[-\alpha-(\alpha+2)]^{n}}{2(\alpha+2)} \\
& =\frac{2}{(2 \lambda)^{n}}\left[n(-\alpha)^{n-1}+\frac{n(n-1)(n-2)}{3 !}(-\alpha)^{n-3}(\alpha+2)^{2}+\cdots\right] \\
& \geqq \frac{2}{(2 \lambda)^{n}}\left[n(-\alpha)^{n-1}+\frac{n(n-1)(n-2)}{3 !}(-\alpha)^{n-3}\left(\alpha^{2}-4 \lambda\right)+\cdots\right] \\
& =\frac{2}{(2 \lambda)^{n}\left(\alpha^{2}-4 \lambda\right)^{1 / 2}}\left[n(-\alpha)^{n-1}\left(\alpha^{2}-4 \lambda\right)^{1 / 2}\right. \\
& \left.\quad+\frac{n(n-1)(n-2)}{3 !}(-\alpha)^{n-3}\left(\alpha^{2}-4 \lambda\right)^{3 / 2}+\cdots\right] \\
& =\frac{1}{\left(\alpha^{2}-4 \lambda\right)^{1 / 2}} \frac{\left(-\alpha+\left(\alpha^{2}-4 \lambda\right)^{1 / 2}\right)^{n}-\left(-\alpha-\left(\alpha^{2}-4 \lambda\right)^{1 / 2}\right)^{n}}{(2 \lambda)^{n}} \\
& =\frac{\left|a_{n}\right|}{\left|a_{-1}\right|} .
\end{aligned}
$$

Combining this with (65), we get $(-\alpha-1) / \lambda>\left|a_{n}\right| /\left|a_{-1}\right|$ which is (63).

This proves (55) which completes Example i. 
The following examples can be demonstrated with approximately the same method used in Example i, although each proof contains its own little difficulty. We present only the results here, and refer the reader to the first author's doctoral thesis for the complete proofs.

EXAMPLE ii. We let $\alpha=-2, \beta=-1$, and we consider only positive $\lambda$. We find that for $\sqrt{ } 2<\lambda \leqq 3 / 2$, (4) holds but $\lambda \in H_{K}$.

EXAMPLE iii. We let $\alpha=-1, \beta=-2$, and again we consider only positive $\lambda$. If we let $\lambda_{0}$ be the zero of $x^{6}-24 x^{2}+16 x-2$ which lies in the interval $(2.01,2.02)$, we find that for $\lambda_{0}<\lambda \leqq 33 / 16$, (4) holds but $\lambda \in H_{K}$.

EXAMPLE iv. We let $\alpha=-1, \beta=-1$, and once again we consider only positive $\lambda$. We find that for $1<\lambda \leqq 9 / 8$, (4) holds but $\lambda \in H_{K}$.

In the proof of Example iv, the following inequality is crucial:

$$
|\sin n \theta|<\frac{\sin \theta}{(2 \cos \theta)^{n+1}} \text { for } \frac{\pi}{3}<\theta<\frac{\pi}{2} \text { and } n \geqq 1 \text {. }
$$

Although a proof for (66) is given in the first author's doctoral thesis, it is rather tedious, and a nicer proof of this elementary inequality would be appreciated.

\section{BiBLIOGRAPHY}

1. R. R. Goldberg, Fourier transforms, Cambridge Univ. Press, Cambridge, 1961.

2. E. Hewitt and K. A. Ross, Abstract harmonic analysis, Vol. 1, Academic Press, New York, 1963.

3. D. J. Newman, Uniqueness theorems for convolution-type equations, Proc. Amer. Math. Soc. 16 (1965), 629-634.

4. W. Rudin, Fourier analysis on groups, Interscience, New York, 1962.

NAVAL Research Laboratory, WASHINGTON, D. C.

YeSHIVA UNIVERSITY,

New York, New York 